\title{
¿Por qué Somos Infieles? Aplicación Inicial de una Escala para Estimar las Razones por las que Hombres y Mujeres son Infieles
}

\author{
Why Are We Unfaithful? Initial Application of a Scale to Estimate the Reasons why \\ Men and Women Are Unfaithful
}

\author{
Oriana Figueroa Valdebenito ${ }^{1,2}$, Ana María Fernández Tapia ${ }^{1}$ y Carolina Jara Durán ${ }^{1}$
}

\begin{abstract}
Resumen
Se presenta la construcción de una escala para estimar razones por las que hombres y mujeres son infieles, tomando como base teórica la perspectiva evolucionista. La Escala de Razones para la Infidelidad (RPI) fue aplicada a una muestra de 311 estudiantes de pre y post grado de la Universidad de Santiago de Chile. Los resultados indicaron una fiabilidad apropiada para la muestra total y una estabilidad temporal moderada a 40 días de la primera aplicación. De acuerdo con un AFE, se configuraron dos dimensiones a partir de los 12 ítems de la escala: insatisfacción con la relación y sexualidad. Como evidencia de validez convergente, se observó una asociación entre la sexualidad y la sociosexualidad y una correlación negativa entre sexualidad del RPI y la calidad de la relación. Se observaron diferencias por género en la dimensión de sexualidad, lo cual concuerda con las predicciones a la base de la investigación.
\end{abstract}

Palabras clave: infidelidad, instrumento, Escala de Razones para la Infidelidad (RPI), psicología evolucionista

\begin{abstract}
This research aimed at generating a scale about the reasons for infidelity in men and women, from an evolutionary perspective. The Reasons for Infidelity Scale (RPI) was administered to 311 undergraduate and graduate students from Universidad de Santiago de Chile. The results exhibited acceptable internal consistency and moderate temporal stability 40 days after the initial application. An exploratory factor analysis resulted in two dimensions to account for the 12 items in the final scale: relationship dissatisfaction and sexuality. A positive association of the dimension of sexuality of the RPI and sociosexuality, as well as a negative correlation of the dimension of sexuality of the RPI and relationship quality evidenced the convergent validity of the scale. Sex differences were observed in the dimension of sexuality. These results are consistent with the predictions in which the study is based.
\end{abstract}

Keywords: infidelity, instrument, Reasons for Infidelity Scale, evolutionary psychology

\footnotetext{
${ }^{1}$ Escuela de Psicología, Universidad de Santiago de Chile, Chile. Correspondencia: oriana.figueroav@gmail.com

${ }^{2}$ Centro de Investigación en Complejidad Social, Universidad del Desarrollo, Santiago, Chile.
} 


\section{Introducción}

La psicología evolucionista se centra en explicar las características de los procesos psicológicos que han sido influenciados fuertemente por contingencias históricobiológicas que la especie ha debido enfrentar en los miles de años de evolución (Cosmides y Tooby, 1997). Dentro de estos, Darwin plantea dos grandes principios evolutivos que regirían la conducta y patrones psicológicos actuales. El primero es conocido como selección natural, que refiere al mecanismo de evolución que favorece la supervivencia de los organismos que logran sobrevivir y reproducirse en un medio hostil, llevando a la extinción a aquellos organismos que no logran tal adaptación (Fernández, 2009).

En tanto, el segundo principio se denomina selección sexual y aborda la evolución de una característica, debido a sus beneficios en la atracción de una pareja favoreciendo una mayor tasa reproductiva y no necesariamente beneficios en términos de supervivencia (Buss, 1994a) y desde donde se desprenderían las reacciones diferenciadas en ambos sexos frente a la conducta de infidelidad de la pareja, tal como se plantea en la teoría de las estrategias sexuales (Buss y Schmitt, 1993; Symons, 1979).

Buss y Schmitt (1993) sostienen que tanto hombres como mujeres han desarrollado a lo largo del tiempo un repertorio de diversas alternativas de emparejamiento, entre las que se incorporan tanto las relaciones estables o de largo plazo como las de corta duración. En relación con el principio de selección sexual y la teoría de las estrategias sexuales, se encuentran costos adaptativos sexualmente dimórficos que permitirían inferir distintos desafíos evolutivos y estrategias de afrontamiento sexualmente diferenciadas en la psicología que subyace nuestra especie (Symons, 1979; Buss 1994a, Buss, 2013).

Así, a la hora de reproducirse, la mujer invierte recursos biológicos costosos, que implican un largo periodo de gestación y sus consecuentes desventajas adaptativas respecto al costo de oportunidad. Por otro lado, la inversión del hombre es menos perceptible en recursos directos, pues este se orientaría al acceso a un número importante de mujeres con el objetivo de preservar sus genes (Easton, Goetz, \& Buss,
2015), pero sería altísima la inversión parental prolongada, en cuidados y recursos asociados a la crianza de un hijo (Buss, 1994b; Fernández, 2009; Buss, 2013).

Las diferencias sexuales que se extraen de lo anterior, tal como lo planteó Trivers (1972), derivan en un marcado dimorfismo sexual en la psicología del hombre en comparación con la mujer, que conlleva a la preferencia masculina por el atractivo físico, la fertilidad y signos de fidelidad sexual de la pareja (signos que maximizan la inversión masculina y aseguran la paternidad); y la preferencia femenina por parejas con un alto potencial financiero y status (influenciado por el propósito de asegurar el bienestar y protección de los hijos y familia), lo que se reflejaría en la incidencia de la infidelidad - entendida como la violación al compromiso de exclusividad con la pareja- y sus consecuentes razones o motivos (Easton et al., 2015; Zuhal \& Kökdemir, 2006).

En torno a la infidelidad en específico es que se ha estudiado como hombres y mujeres han desarrollado algunas estrategias que han permitido la retención de la pareja. En este sentido, los celos aparecen como una estrategia desarrollada por nuestros ancestros como una forma de proteger un vínculo emocional valorado ante un posible escenario de infidelidad emocional o sexual (Buunk, 1997). Dicha estrategia fue impulsada como una forma de mantener una suerte de estabilidad sexual y emocional, esperada idealmente por toda unión en pareja, entendiendo que la monogamia no es la conducta "naturalmente evolutiva" de la especie humana y que la fidelidad de la pareja sólo se afirmaría en la decisión mutua y consciente de mantener este vínculo de forma exclusiva con una sola persona (Fisher, 1992).

De ahí, es que la noción de infidelidad aparece como la violación al compromiso de exclusividad emocional y sexual con la pareja y ha sido estudiada en diferentes poblaciones, incluyendo a estudiantes universitarios, terapias de pareja y muestras comunitarias, en las cuales se han desarrollado variados métodos de investigación como auto informes, recolección de experiencias personales de infidelidad y estudiando las reacciones que tienen las personas frente a viñetas hipotéticas de infidelidad, entre 
otros (Allen \& Baucom 2006; Edlund \& Sagarin, 2017).

La noción de fidelidad se entiende como la decisión de compartir tanto los recursos físicos como emocionales exclusivamente con otro, por lo que se encuentra altamente relacionada al concepto de satisfacción y compromiso percibido con la relación, en donde es importante la reciprocidad que cada miembro de la pareja perciba en la relación (Ojeda, Torres, \& Moreira, 2010).

Dentro de este contexto, pero incorporando una mirada desde la psicología social, Laumann (1994), plantea que un tercio de los hombres y un quinto de las mujeres casadas han tenido sexo extramarital. Sin embargo, se señala que la mayoría de los estadounidenses desaprueban las relaciones extramatrimoniales (Schmitt \& Buss, 2001) y en los estudios en nuestra región, se ha encontrado diversidad de formas de conceptualizar las actitudes hacia la sexualidad (Rojas Tejada y Blanc Molina, 2017).

En este escenario, un estudio que incluyó 63 países de Schmitt y sus colaboradores (2004), encontraron que alrededor de un $60 \%$ de los hombres y un $40 \%$ de las mujeres reconoce haber seducido a alguien comprometido al menos una vez en la vida. Asimismo, un $80 \%$ de dicha muestra ha estado involucrada en una relación de este tipo, ya sea como seductor, seducido o pareja del seducido. Estas cifras fueron comparables y estables según los datos de una investigación local en nuestro país (Barahona, 2006). Y la prevalencia de la apertura a relaciones sexuales sin compromiso en jóvenes chilenos, tiene una preponderancia en hombres dos veces más alta que en mujeres (Fernández, Celis-Atenas, Córdova-Rubio, Dufey, Corrêa Varella, \& Benedetti Piccoli Ferreira, 2013).

Por otra parte, también se ha comprobado en países de habla hispana (García-Leiva, GómezJacinto, \& Canto, 2001), alguna preferencia por las características que buscan hombres y mujeres en una posible pareja, rasgos que podrían contribuir a dilucidar factores que estarían a la base de la conducta de infidelidad, donde las características de la pareja más valoradas en una relación estable serían: carácter agradable, inteligencia, sinceridad y fidelidad sexual. Asimismo, el atractivo físico y la juventud se valoran más en la elección de los hombres que en la de las mujeres, siendo esto comparable con los hallazgos internacionales de Buss $(1989 ; 2013)$ y recientemente de Puts (2010).

Para el presente estudio, se recurrió a los postulados evolucionistas, con el fin de describir la génesis, frecuencia y mantención de la conducta de infidelidad dentro de las parejas, los que proponen la existencia de diferencias psicológicas entre ambos sexos frente a las distintas estrategias reproductivas utilizadas en la consecución de esta conducta.

Se tomó como referencia para la construcción inicial de los ítems de la escala, un estudio realizado en Turquía por Zuhal y Kökdemir (2006), que conceptualiza las razones para la infidelidad y busca diferencias sexuales en seis dimensiones que aluden a las diferencias entre hombres y mujeres y también a factores culturales, tales como los matrimonios "arreglados" o las diferencias en torno a la sanción social frente a la infidelidad de la pareja.

Para la construcción preliminar de la escala se tomaron como referencia las dimensiones que aluden tanto a componentes emocionales y sexuales que llevarían a cometer infidelidad, como también aquellos que tienen que ver con las características del rival y la elección de la infidelidad como un comportamiento válido dentro de la relación (Zuhal \& Kökdemir, 2006).

En la presente investigación se construyó un instrumento sobre las razones por las que hombres y mujeres son o serían infieles, donde al igual que en el estudio revisado anteriormente, no tuvo importancia si el sujeto fue o es infiel, pues el énfasis estuvo en las posibles causas de esta conducta, independientemente de su ocurrencia en el pasado o el presente. La relevancia del estudio, no sólo es a nivel metodológico, al desarrollar una escala para medir y sistematizar los motivos que se dan en la conducta de infidelidad, sino que también es a nivel teórico, debido a que se realizó una primera estimación desde la perspectiva evolucionista de aquellos factores y/o dimensiones que se encuentran a la base de tal conducta, en el contexto chileno.

El objetivo de la investigación fue construir de un instrumento que describa de manera confiable y válida las posibles razones que motivarían la conducta de infidelidad. Para luego 
indagar las diferencias sexuales que se correspondan con la teoría de las estrategias sexuales postuladas desde la Psicología Evolucionista (Buss, 2013).

Con este propósito se conceptualizó la infidelidad como la transgresión al compromiso de exclusividad emocional y sexual que se encuentra dentro de una relación de pareja (García-Leiva et al., 2001). Buscando responder ¿Cuáles son las posibles razones por las que hombres y mujeres son infieles?; y, por otra parte, si ¿Existen diferencias sexuales en las razones o motivos para la conducta de infidelidad?

\section{Método}

\section{Participantes}

La muestra final fue de tipo no probabilístico intencional y estuvo conformada por 311 estudiantes universitarios de pre y post grado de la Universidad de Santiago de Chile, excluyendo a la carrera de psicología, cuyas edades fluctuaron entre los 18 y 59 años $(M=21.9, D T=6.16)$, siendo el $48.2 \%$ mujeres y el $48.8 \%$ varones. El $93.1 \%$ reporta ser heterosexual, $2.5 \%$ homosexual, $2.5 \%$ bisexual, un $1.2 \%$ no reportó su orientación sexual. Además del total de la muestra el $90.6 \%$ reporta no tener hijos, mientras que el $9.1 \%$ tiene entre 1 y 4 hijos.

En relación a la situación sentimental de los encuestados, un $25.6 \%$ de hombres y un $31.2 \%$ de las mujeres, se encontraba saliendo con una sola persona, mientras que $39.1 \%$ de hombres y $37 \%$ de mujeres no tenían pareja.

\section{Construcción del Instrumento}

En la primera etapa de la investigación, se realizó una revisión de la literatura, en función de la cual se tomó como eje la perspectiva evolucionista, con el objetivo de definir la infidelidad y la búsqueda de ciertas dimensiones posibles de incluir en la construcción del instrumento. De acuerdo a los lineamientos evolucionarios y la teoría de las estrategias sexuales (Buss \& Schmitt, 1993), fue posible predecir que en el constructo a estimar existirían diferencias sexuales, lo cual se vería reflejado en la frecuencia de aparición de determinadas razones o motivos para la infidelidad en hombres y mujeres, tal como se encontraron en el estudio realizado por Zuhal y Kökdemir (2006) cuyas dimensiones y preguntas se utilizaron como referente para la construcción de los ítems de la Escala de Razones para la Infidelidad (RPI), las cuales fueron organizadas en seis dimensiones diferenciadas por sexo.

Se construyeron 40 ítems, los que fueron sometidos a un examen subjetivo por 10 jueces expertos (psiquiatras, psicólogos, metodólogos y terapeutas de pareja), quienes sugirieron eliminar 23 ítems debido a que su contenido no se acercaba al constructo evaluado. Con los 17 ítems restantes se elaboró la escala final, utilizando un formato de respuesta en escala Likert de 1 (completamente en desacuerdo) a 7 (completamente en desacuerdo). Para la aplicación piloto del instrumento se tomó contacto con los participantes a través de los profesores de las carrera de pre y pos grado de la Universidad de Santiago de Chile a quienes se les entregó una copia de la escala RPI, el instrumento SOI y PRQC, además de una encuesta de datos sociodemográficos y el consentimiento informado en donde se consignaba la posibilidad de abandonar la participación en cualquier momento, así como también la confidencialidad de la información y el uso de los datos con fines académicos. En esta etapa de la construcción del instrumento participaron 79 estudiantes de pre y pos grado siendo el $62 \%$ mujeres y $34.2 \%$ varones, cuyas edades fluctuaron entre los 18 y 59 años, $(M=30.73, D T=6.16)$. En relación a la situación sentimental de los encuestados $5.1 \%$ reporta estar casado, $16.5 \%$ comprometido, el $2.5 \%$ conviviendo, $1.3 \%$ saliendo con varias personas, $27.8 \%$ saliendo con una persona, $40.5 \%$ sin pareja en la actualidad, $5.1 \%$ nunca ha tenido una relación sexual. Mientras que el $35.4 \%$ ha sido infiel y el $41.8 \%$ reportó que alguna pareja le había sido infiel.

Dado el adecuado tamaño de la muestra utilizado ( $n=79)$ la medida de adecuación muestral de Kaiser-Meyer-Olkin es propicia (.58) para proceder a realizar un análisis factorial (Índice de Esfericidad de Bartlett $\chi 2=386.14, p<.000)$. Pese al bajo tamaño muestral, el análisis exploratorio arrojó cinco factores en función del criterio de normalización de Kaiser y con rotación Varimax. Los cinco factores explican el $61.51 \%$ de la varianza total. La primera dimensión, explica el $21.78 \%$ de la varianza total, la segunda un 
$14.79 \%$, la tercera un $9.96 \%$, la cuarta un $8.15 \%$ y la quinta un $6.8 \%$.

Las dimensiones fueron construidas de la siguiente forma:

Insatisfacción con la Relación: Refiere a las causas que llevarían a desarrollar la conducta de infidelidad y que tienen que ver principalmente con la falta de compromiso percibida, la falta de sensibilidad, o tener proyectos de vida distintos a los de la pareja.

Sexualidad: Alude a la satisfacción y el rendimiento sexual de la pareja, además de la presencia de tabúes sexuales dentro de la relación, lo cual de alguna manera podría identificarse como una posible razón para desarrollar la conducta de infidelidad.

Legitimación de la Infidelidad: Incluye los ítems que refieren a percibir el acto de infidelidad como un juego o como parte de la vida en pareja, lo cual de alguna forma se encuentra mediado por aspectos culturales.

Apertura Sociosexual: Refiere a la apertura individual a tener relaciones y fantasías sexuales fuera de la relación de pareja o a ejercer la conducta de infidelidad frente a la infidelidad del compañero.

Constancia y Estabilidad Emocional: Alude a la constancia dentro de la relación, en términos de proyección y estabilidad y exclusividad del vínculo con la pareja.

Se utilizó una carga factorial de .30 para retener los ítems en cada factor.

Los resultados indican una buena fiabilidad (coeficiente alfa de Cronbach=.74) para la escala total, donde los cinco factores extraídos contienen buenos niveles de confiabilidad, por lo que se conservan todos los ítems para su aplicación final.

\section{Instrumentos}

Para evaluar la validez de la RPI se utilizaron dos instrumentos adicionales.

Inventario de Orientación Sociosexual. (SOI, Simpson \& Gangestad, 1991), mide la apertura individual a las relaciones sexuales, y debiese relacionarse positivamente con las razones 0 motivaciones para la infidelidad. En cuanto a la consistencia interna del instrumento ésta ha alcanzado un $\alpha$ de Cronbach $=.80$ y ha mostrado validez discriminante entre hombres y mujeres, y validez convergente con otros constructos (e.g., celos; ver estudios de Fernández, 2008; Fernández, et al., 2013).

Inventario de Componentes de la Calidad de las Relaciones. (PRQC, Fletcher, Simpson, \& Thomas, 2000) mideaspectos de satisfacción con la relación (i.e., la satisfacción, el compromiso, la intimidad, confianza, pasión y el amor dentro de la relación) y se espera que correlacione negativamente con la RPI. En Chile, estuvo adaptado por el International Sexuality Description Project (Schmitt et al., 2004), el cual disminuyó a la mitad el cuestionario original de Fletcher, et al., (2000), comprobando su relación y equivalencia con los resultados de una aplicación en más de 60 países y su consistencia interna alcanzó un coeficiente $\alpha$ de Cronbach $=.91$.

\section{Análisis de datos}

Para el análisis final de la escala, se realizó un análisis factorial exploratorio (AFE) con extracción por ejes principales, rotación oblicua (promax), y análisis paralelo de Horn (cf., Dinno, 2009) para la retención de factores. Esto con la finalidad de utilizar un criterio concluyente para la estructura dimensional subyacente al constructo. Luego, se evaluó su validez concurrente y divergente, mediante el análisis de correlaciones entre el RPI y los instrumentos de criterio.

Adicionalmente, se evaluó la fiabilidad del instrumento mediante dos procedimientos independientes: su estabilidad temporal (coeficiente de correlación $r$ de Pearson entre la pre y postprueba 40 días después de la primera aplicación) y como consistencia interna (coeficiente $\alpha$ de Cronbach). Asimismo, se utilizaron estadísticos descriptivos como la media y desviación estándar para explicitar los niveles del constructo en la muestra y se evaluaron diferencias por sexo predichas por la teoría.

Todos los análisis de datos fueron realizados con el paquete estadístico SPSS 21.0 y para la simulación de los datos del análisis paralelo, se utilizó el programa de simulación de Watkins (2008) y se siguió el criterio de Matzunga (2011).

\section{Resultados}

\section{Análisis Factorial Exploratorio}

Según la medida de adecuación muestral de Kaiser-Meyer-Olbimin $(\mathrm{KMO})=.86$, se procedió 
Tabla 1. Resultados del análisis paralelo de Horn para la retención de factores

\begin{tabular}{lccc}
\hline Factor & $\begin{array}{c}\text { Autovalor observado en la } \\
\text { muestra }\end{array}$ & Autovalor esperado por azar & Factores a retener \\
\hline 1 & 5.51 & 1.41 & $\mathrm{Si}$ \\
2 & 2.19 & 1.32 & $\mathrm{Si}$ \\
3 & 1.18 & 1.25 & No \\
\hline
\end{tabular}

Nota. Sólo se retienen los factores cuyo autovalor observado supera el autovalor esperado por azar.

Tabla 2: Conformación Definitiva de la Escala RPI

\begin{tabular}{rlcc}
\hline Ítems & & $\begin{array}{c}\text { Insatisfacción } \\
\text { con la relación }\end{array}$ & Sexualidad \\
\hline 1. No existe proyección con la pareja & .55 & .06 \\
2. La pareja no se compromete realmente con la & $\mathbf{. 7 8}$ & -.02 \\
relación & $\mathbf{. 7 2}$ & .16 \\
3. La pareja no muestra un involucramiento real en la & & \\
relación & $\mathbf{. 7 6}$ & .08 \\
4. Insatisfacción emocional con la pareja & $\mathbf{. 5 2}$ & .11 \\
5. Mantener una relación monótona & $\mathbf{. 6 1}$ & -.27 \\
6. Intereses y proyectos distintos a los de la pareja & $\mathbf{. 7 0}$ & -.21 \\
7. No confiar en la pareja & -.19 & $\mathbf{. 4 1}$ \\
8. La infidelidad es normal dentro de una relación & -.01 & $\mathbf{. 6 6}$ \\
9. Insatisfacción sexual con la pareja & -.00 & $\mathbf{. 5 7}$ \\
10. Presencia de tabúes sexuales en la relación & -.07 & $\mathbf{. 6 6}$ \\
11. Disminución del rendimiento sexual del & .17 & $\mathbf{. 7 7}$ \\
compañero/a & 12. Falta de empatía de la pareja en el ámbito sexual &
\end{tabular}

Nota. Los valores marcados en negrita son las cargas de los ítems correspondientes a cada factor

a realizar el AFE con análisis paralelo. Como se muestra en la Tabla 1, son dos el número de factores con capacidad explicativa mayor que el azar.

Estos dos factores explicaron el $45.37 \%$ de la varianza total (la primera dimensión $32.46 \%$, la segunda un $12.91 \%)$. Así, se generaron dos dimensiones con el siguiente significado:

Insatisfacción con la Relación: Refiere a las causas que llevarían a desarrollar la conducta de infidelidad como la falta de proyección y compromiso percibida, falta de sensibilidad, o tener proyectos de vida distintos a los de la pareja.

Sexualidad: Alude a la satisfacción y el rendimiento sexual de la pareja, además de la presencia de tabúes sexuales dentro de la relación.

La correlación entre las dimensiones de Insatisfacción con la relación y Sexualidad resultó ser de $r=.57$.

\section{Fiabilidad}

Los resultados mostraron valores aceptables de consistencia interna: en Insatisfacción con la relación ( $\alpha$ de Cronbach $=.84$, con siete ítems) y Sexualidad ( $\alpha$ de Cronbach=.77, con cinco ítems).

Después de 40 días de la primera aplicación, se aplicó nuevamente la escala a 30 participantes que accedieron a contestarla voluntariamente, observándose una correlación positiva y moderada, estadísticamente significativa $(r=.51$, $p<.002)$ entre la primera y segunda aplicación.

\section{Validez}

La validez convergente fue evaluada en la aplicación definitiva del instrumento, correlacionando las dos dimensiones de la escala RPI con las variables criterio.

Se encontró una correlación positiva pero baja entre SOI y la dimensión de sexualidad $(r=.28$, 
Tabla 3. Diferencias por sexo en el puntaje promedio de las dimensiones del RPI

\begin{tabular}{lcccc}
\hline Dimensión & Hombre & Mujer & $t$ & $d$ de Cohen \\
\hline Insatisfacción con la relación & $4.64(1.28)$ & $4.58(1.56)$ & 0.41 & .04 \\
Sexualidad & $3.81(1.20)$ & $3.37(1.29)$ & $3.10^{*}$ & .35 \\
\hline
\end{tabular}

Nota. $n=311 . * p<.01$

$p<.001)$. A su vez, se encontró una correlación negativa y baja entre el PRQC y la dimensión sexualidad del RPI $(r=-.26, p=.000)$, en la dirección esperada teóricamente.

Se encontraron diferencias estadísticamente significativas en la dimensión de sexualidad del RPI, observándose un puntaje más alto en los hombres respecto de las razones o motivos para la infidelidad que las mujeres (ver Tabla 3), tal como se hipotetizaba.

\section{Discusión}

El presente estudio, se planteó como una forma de aportar conocimientos desde la psicología evolucionista en relación a las posibles razones o motivos por los cuales hombres y mujeres son infieles, desde donde se construyó una escala que determina estas razones y que se agruparon en dos factores: insatisfacción con la relación y sexualidad, los que resultan coherentes con los planteamientos de la psicología evolucionista que plantea a ambas dimensiones como de importancia para los sexos a partir de la teoría de las estrategias sexuales.

El instrumento Escala de Razones para la Infidelidad, logra una aceptable fiabilidad por consistencia interna y validez convergente para estimar cuales son las posibles razones por las que hombres y mujeres son infieles. Además, se estima un nivel moderado de estabilidad temporal del instrumento, lo cual fue corroborado a través del método de prueba y postprueba.

Asimismo, se constataron diferencias en las razones o motivos para la infidelidad entre hombres y mujeres correspondiéndose con la teoría de las estrategias sexuales, donde los hombres tenderían a puntuar más alto en las razones de índole sexual lo que de acuerdo a los postulados evolucionistas, tendría mayor importancia en la relación de pareja y en la búsqueda de una compañera fuera de la relación; pues motiva la maximización de oportunidades reproductivas. Mientras que las mujeres tenderían a ponderar más alto las razones de índole emocional, donde la búsqueda del compañero se encontraría motivada a factores de seguridad y estabilidad emocional que aseguren el bienestar propio y de los hijos (Easton et al., 2015).

Respecto a la calidad de las relaciones y la dimensión de sexualidad del RPI, se encuentran relaciones inversas, es decir, a mayor calidad de las relaciones existiría una menor probabilidad de desarrollar la conducta de infidelidad, no existiendo diferencias sexuales en esta asociación, pero mostrando una baja magnitud. De la misma forma, existiría una relación positiva en cuanto a la apertura sociosexual y la dimensión de sexualidad del RPI, es decir, mientras mayor apertura individual a las relaciones sexuales sin compromiso, mayor probabilidad de desarrollar la conducta de infidelidad relacionada con carencias en sexualidad.

En cuanto al componente valórico y cultural, es posible mencionar que en comparación con el instrumento turco la construcción de la escala RPI, excluyó temas culturales y valóricoreligiosos, donde sólo se aprecia claramente la validación social que existe respecto de la infidelidad de los hombres, lo cual quedó plasmado en la escala RPI (Zuhal \& Kökdemir, 2006).

Además, de acuerdo a las dimensiones de cada instrumento es posible establecer el sesgo que marcaba la diferencia cultural entre ambos, pues la distribución de los ítems en las distintas dimensiones planteadas, diferían en su significado para la población chilena, por lo que se hacía muy difícil su adaptación en este contexto.

En cuanto a las limitaciones del presente estudio, la evidencia de validez mostrada debe ser considerada como preliminar, debido a que la magnitud de los valores estadísticos de convergencia es baja.

Como proyecciones se plantea la necesidad de ampliar la muestra a población general de manera de observar el comportamiento de las propiedades métricas de la escala en una muestra distinta de la 
estudiada, con el objetivo de corroborar la estructura bi-dimensional de la escala RPI. Asimismo, para investigaciones posteriores se podría indagar en otros aspectos relevantes desde lo evolucionista y social como aspectos que debiesen ser incluidos en el desarrollo de nuevas escalas tales como los roles sexuales, fantasías sexuales, la percepción ante la infidelidad, el papel de los celos, entre otras.

\section{Referencias}

Allen, E. S., \& Baucom, D. H. (2006). Dating, marital, and hypothetical extradyadic involvements: How do they compare. Journal of Sex Research, 43, 307-317. doi:10.1080/00224490609552330

Baraona, M. (2006). La tendencia a seducir a lguien que se encuentra comprometido: ¿Función evolutiva o actitud aprendida?. Estudio experimental realizado con una muestra de estudiantes universitarios. Tesis para optar al titulo de psicólogo clínico. Universidad de Santiago de Chile. Santiago. Chile.

Buss, D. M. (1989). Sex differences in human mate preferences: Evolutionary hypotheses tested in 37 cultures. Behavioral and Brain Sciences, $12, \quad 1-49$. doi: 10.1017/S0140525X00023992

Buss, D. M. (1994a). The strategies of human mating. American Scientist, 82, 238-249. Recuperado de: http://labs.la.utexas.edu/buss/files/2015/09/A merSciMay 1994.pdf

Buss, D. M. (1994b). La evolución del deseo: Estrategias del emparejamiento humano. Madrid: Alianza Editorial. ISBN 9788491040828

Buss, D. M. (2013). The science of human mating strategies: An historical perspective. Psychological inquiry: An international journal for the advancement of psychological theory, 24, 171-177. doi:10.1080/1047840X.2013.819552

Buss, D. M., \& Schmitt, D. P. (1993). Sexual strategies theory: an evolutionary perspective on human mating. Psychological Review, 100, 201-232. doi:10.1037/0033295X.100.2.204
Buunk, A. P. (1997). Personality, birth order and attachment styles as related to various types of jealousy. Personality \& Individual Differences, 23, 997-1006. doi:10.1016/s0191-8869(97)00136-0

Cosmides, L. \& Tooby, J. (1997). Evolutionary psychologist: A primer. Center for Evolutionary Psychologist, Santa Bárbara. Recuperado de: http://www.cep.ucsb.edu/primer.htmlhttp://w ww.cep.ucsb.edu/primer.html

Easton, J. A., Goetz, C.D., \& Buss, D. M., (2015). Human mate choice, evolution of. In: James D. Wright (editor-in-chief), International Encyclopedia of the Social \& Behavioral Sciences, 340-347, $2^{\text {nd }}$ ed, Vol 11. Oxford: Elsevier.

Edlund, J. E., \& Sagarin, B. J. (2017). Sex differences in jealousy. Advances in Experimental Social Psychology, 259-302. doi:10.1016/bs.aesp.2016.10.004

Fernández, A. M. (2008). Seducción, celos y sociosexualidad en estudiantes universitarios: Resultados preliminares. En I. Magaña, Encuentros y Desafios de la Investigación en Ciencias Sociales y Humanidades, 29-56. Santiago: PUBLIFAHU-USACH.

Fernández, A. M. (2009). Psicología evolucionaria: Un marco integrador para la disciplina psicológica en el bicentenario de Darwin. Revista de Psicología Universidad de Chile, 18, 9-24. doi: 10.5354/07190581.2009.17118

Fernández, A. M., Celis-Atenas, K., CórdovaRubio, N., Dufey, M., Corrêa Varella, M. A., \& Benedetti Piccoli Ferreira, J. H. (2013). Sexualidad juvenil: Prácticas, actitudes y diferencias según sexo y variables de personalidad en universitarios chilenos. Revista Médica de Chile, 141, 160166.doi:10.4067/s0034-98872013000200003

Fisher, H. E. (1992). The Anatomy of Love. New York. Ballantine Books.

Fletcher, G. Simpson, J. y Thomas, G. (2000). The measurement of perceived relationship quality components: A confirmatory factor analytic approach. Personality and Social Psychology Bulletin, 26, 340-354. doi:10.1177/0146167200265007 
García, P. Jacinto, L., \& Canto, J. (2001) Reacción de los celos ante una infidelidad: diferencias entre hombres y mujeres y características del rival. Psicothema, 13,611616. ISSN 0214-9915

Laumann, E. O. (1994). The social organization of sexuality: Sexual practices in the United States. Chicago: University of Chicago Press.

Matzunaga, R. (2011). How to factor analyze your data right: Dos, don'ts and how-tos. International Journal of Psychological Research, 3, 97-110. doi:10.21500/20112084.854

Ojeda, A., Torres, G., \& Moreira, M., (2010). ¿Amor y compromiso en la pareja? de la teoría a la práctica. Revista Iberoamericana de Diagnóstico y Evaluación - e Avaliação Psicológica, 30, 125-142. Recuperado de: http://www.redalyc.org/pdf/4596/4596454420 08.pdf

Puts, D. A. (2010). Beauty and the beast: Mechanisms of sexual selection in humans. Evolution and human behavior, 31, 157-175. doi: 10.1016/j.evolhumbehav.2010.02.005

Rojas Tejada, A. J., \& Blanc Molina, A. (2017). Instrumentos de Medida de Actitudes hacia la Sexualidad: Una Revisión Bibliográfica Sistemática. Revista Iberoamericana de Diagnóstico y Evaluación - e Avaliação Psicológica, $\quad 43, \quad 17-32$. doi:10.21865/ridep43_17

Schmitt, D. P., \& 121 Members of International Sexuality Description Proyect. (2004). ¿Patterns and universals of adult romantic attachment across 62 cultural regions. Are models of self and of other pancultural constructs?. Journal of Cross Cultural Psychology, 35, 367-402. doi:10.1177/0022022104266105

Schmitt, D. P., \& Buss, D. M. (2001). Human mate poaching: Tactics and temptations for infiltrating existing mateships. Journal of personality and social psychology, 80, 894917. doi:10.1037//0022-3514.80.6.894

Simpson, J. A., \& Gangestad, S. W. (1991). Individual differences in sociosexuality: Evidence for convergent and discriminant validity. Journal of Personality and Social Psychology, 60, 870-883. doi:10.1037/00223514.60.6.870
Symons, D. (1979). The evolution of human sexuality. New York: Oxford University Press.

Trivers, R. (1972). Parental Investment and Sexual Selection. En B.Campbell (ed.), Sexual Selection and the Descent of Man (p. 136179). New York: Aldine \& Gruyter.

Zuhal,Y. y Kökdemir, D. (2006). University student's perceptions of and explanations for infidelity: The development of the infidelity questionnaire (INFQ). Social Behavior and Personality, 34, 639-650. doi: 10.2224/sbp.2006.34.6.639 